\section{Supernova examined by computer model}

THE uncertainty discussed here last week as to whether Sanduleak (Sk)-69 202 was the progenitor of supernova SN 1987A continues unresolved. Dave Arnettt of the University of Chicago will no doubt be watching developments closely. He has been using computer models of stellar evolution to find out what sort of explosion would best explain the various observations - in particular its faintness and unusual light curve - and by thus working backwards his theoretical candidate for the supernova progenitor is a blue supergiant within about half a magnitude of Sk-69 202.

Arnett's success removes several difficulties. A standard type II supernova explosion was excluded for at least three reasons. Its progenitor, a red supergiant, would have been visible on optical plates of the region; the outburst would have been several magnitudes brighter than SN 1987A; and the time interval between the neutrino pulse and the optical sighting would have been longer than was the case.

These problems indicated that a smaller, fainter star would make a better progenitor. (Neutrinos emerge promptly from the collapsed core, but photons must fight their way out through the dense surrounding material; a smaller progenitor has, for this simple reason, a faster optical rise time.) But a smaller star would generate insufficient ${ }^{56} \mathrm{Ni}$, whose radioactive decay is thought to power the recently observed brightening of the expanding shell. So some other source, perhaps a pulsar, would have to be adduced. Things were becoming complicated, and unconvincing.

Arnett's model is pleasingly straightforward. He takes a 15-solar mass star, gives it element abundances appropriate to the Large Magellanic Cloud (LMC), and lets it evolve. Lower elemental abundances compared with our Galaxy make the crucial difference; the central nuclear reactions are less efficient, so the star has to be hotter and denser, and therefore smaller, to maintain radiation pressure against gravitational collapse. Equally, the star is more prone to core collapse, and explodes as a less highly evolved blue star, not as an old red giant. And as a bonus, this massive star synthesizes enough ${ }^{56} \mathrm{Ni}$ to explain the later part of the light curve.

Happily, Arnett can also explain why supernovae are rare in irregular dwarf galaxies such as the LMC. Low element abundances are characteristic of these galaxies, so all 'type II' supernovae are fainter than usual, and we see them less easily.

Although there is still uncertainty over the identification of the progenitor, things are looking clearer - at least for this week. David Lindley

Superconducting ceramics On the way to a new technology

\author{
Noboru Miura
}

SinCE the recent discovery of a high-temperature superconductor with the transition temperature, $T_{\mathrm{c}}$, exceeding $30 \mathrm{~K}$ by Bednorz and Müller of IBM, Zurich, and the subsequent experiments by $S$. Tanaka's group in Tokyo last year, tremendous efforts have been made by scientists throughout the world, in particular to achieve even higher transition temperatures. $T_{c}$ now exceeds $90 \mathrm{~K}$, well above the boiling point of nitrogen. The new materials are oxide ceramics, a completely new class of superconductors. Superconductors carry electric currents with no energy loss, so that they are ideal materials for making high-field magnets as used in magnetohydrodynamic generators, accelerators, nuclear fusion reactors, magnetic levitation, energy storage, medical instruments and so on. They could also be used in lossless power transmission lines. The Josephson effect of superconductors can be used to make ultra-fast computers and other electronic devices.

The highest $T_{\mathrm{c}}$ measured previously was $23 \mathrm{~K}$, in a niobium-germanium alloy, which requires the use of very expensive liquid-helium coolant to achieve the superconducting state. Because the new materials superconduct at temperatures obtained by liquid nitrogen, which is much cheaper ( 22 cents per gallon rather than $\$ 11$, many applications are becoming feasible. As pointed out by Professor S. Nakajima (Tokai University) this discovery is comparable to, or even greater than, the invention of the transistor, because it affects not only the field of electronics but also the technology of highpower electricity. It is not an exaggeration to say that such a development will bring about a new industrial revolution, which is why so many people are so excited.

A special session was devoted to the superconductors at the annual meeting of the Physical Society of Japan on 28 March at Nagoya Institute of Technology, attended by more than 1,000 people (see page 432 of last week's issue). In addition to the 18 scheduled talks, 49 prepared discussion papers were accepted just before the session started. The session began at 9.30 a.m. and lasted until 10.40 p.m. The fevered atmosphere seems comparable with that at the American Physical Society Meeting held in New York on 18 March (see the article by M. Strongin et al. on pages 540-541 of last week's issue).

There are three classes of high- $T_{\text {. }}$ ceramic superconductors (S. Tanaka, University of Tokyo): $\mathrm{BaPb}_{1-x} \mathrm{Bi}_{x} \mathrm{O}_{3}$ (a prototype of the newly discovered compounds) with $T=14 \mathrm{~K}$; La-X-Cu-O $(\mathrm{X}=\mathrm{Ba}, \mathrm{Sr}, \mathrm{Ca})$ for which $T_{\mathrm{c}} \sim 40 \mathrm{~K}$; and $\mathrm{Y}-\mathrm{Ba}-\mathrm{Cu}-\mathrm{O}$ for which $T_{c} \sim 90 \mathrm{~K}$. There are many reports claiming different $T_{\mathrm{c}} \mathrm{s}$ in substances with different composition; but for them to be classed as real superconductors, Tanaka insisted on four conditions that had to be satisfied: the structure of the material should be clarified; the Meissner effect should be observed; the electrical resistivity should drop to zero; and the experiments should be reproducible.

The preparation of samples and the measurement of their electrical and magnetic properties were reported by many groups, including that of S. Hikama (University of Tokyo). This group discovered the second class of the new superconductors independently of C.W. Chu of the University of Houston. Agreement on their structure seems to have been obtained between the different groups. The $\mathrm{La}-\mathrm{X}-\mathrm{Cu}-\mathrm{O}$ system has a $\mathrm{K}_{2} \mathrm{NiF}_{4}$ type structure, and the $\mathrm{Y}-\mathrm{Ba}-\mathrm{Cu}-\mathrm{O}$ system has the composition $1: 2: 3: 7-\delta$. For the latter, however, there are still different models for the position of the oxygen atoms. Neutron diffraction reveals a model similar to that of the Bell group (F. Izumi, National Institute for Research on Inorganic Materials). For the first class of superconductors, the structural changes caused by temperature and the barium content $x$ were examined: there are two tetragonal phases for large $x(\mathrm{~K}$. Ohbayashi, Hiroshima University).

The Hartree-Fock instability against spin density waves was examined theoretically as a function of $x$, and the phase diagram constructed (Y. Hasegawa, Institute of Solid State Physics). Such a phase diagram was determined experimentally by magnetic susceptibility measurements (T. Fujita, Hiroshima University). Band calculations were done by $\mathrm{K}$. Takegahara et al. (Tohoku University) using the augmented plane-wave method. Ultraviolet photoelectron spectroscopy data show that the density of states at the Fermi level is rather small ( $T$. Takahashi, Tohoku University). Surprisingly, little change is observed in $T_{\mathrm{c}}$ for the second class of superconductors, even if yttrium is substituted by a series of lanthanide atoms (H. Takagi, University of Tokyo). For the first class, the substitution does not destroy the superconductivity but $T_{\mathrm{c}}$ depends on the substituting lanthanide atom. A neutron-diffraction experiment reveals that there is a spin order in which each copper atom has a magnetic moment of 1.1 $\mu_{\mathrm{B}}$ (Yamaguchi, Tohoku University).

Besides sintered samples, various other 